\title{
GAUGE EQUIVALENCES FOR FOLIATIONS AND PRE-SYMPLECTIC STRUCTURES
}

\author{
FLORIAN SCHÄTZ AND MARCO ZAMBON
}

\begin{abstract}
We consider the deformation theory of two kinds of geometric objects: foliations on one hand, pre-symplectic forms on the other. For each of them, we prove that the geometric notion of equivalence given by isotopies agrees with the algebraic notion of gauge equivalence obtained from the $L_{\infty}$-algebras governing these deformation problems.
\end{abstract}

\section{Contents}

Introduction 1

1. Review: Deformations of foliations and of pre-symplectic structures 2

1.1. Deformations 2

1.2. Foliations and their deformations 3

$\begin{array}{lll}1.3 . & \text { Pre-symplectic structures and their deformations } & 4\end{array}$

\begin{tabular}{llll}
\hline 1.4. & A strict morphism relating deformations & 6
\end{tabular}

2. Equivalences 6

\begin{tabular}{ll}
2.1. & Gauge equivalence \\
\hline
\end{tabular}

\begin{tabular}{lll}
\hline 2.2. & Equivalences of foliations & 7
\end{tabular}

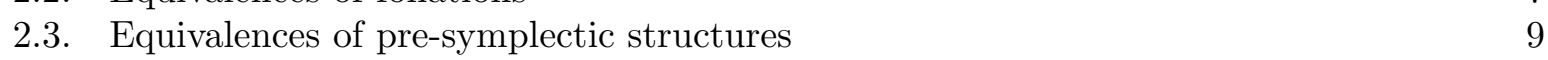

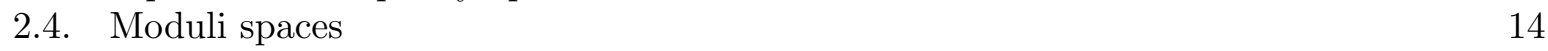

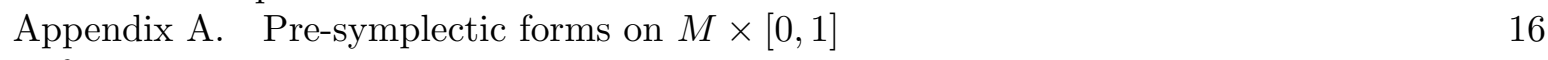

$\begin{array}{ll}\text { References } & 17\end{array}$

\section{INTRODUCTION}

Pre-symplectic forms are closed 2-forms whose kernel has constant rank. They arise for instance by restricting symplectic forms to coisotropic submanifolds, such as the zero level sets of moment maps. They are precisely the 2 -forms which admit local coordinates making them constant, and they can be also regarded as transversally symplectic foliations. The deformation theory of pre-symplectic forms - unlike the one of symplectic forms - is non-trivial, due to the fact that two conditions have to be preserved simultaneously: the closeness condition, and the constant rank one. In a previous publication [17] we showed that pre-symplectic deformations of a pre-symplectic manifold $(M, \eta)$ are governed by an $L_{\infty}$-algebra $L_{\infty}(M, \eta)$ whose brackets are trivial except possibly for those of arity one, two and three.

Further, in [17] we related the $L_{\infty}$-algebra $L_{\infty}(M, \eta)$ to the $L_{\infty}$-algebra governing deformations of involutive distributions (i.e., of foliations), by means of a strict morphism. This can be seen as an enhancement of the geometric fact that to every pre-symplectic form there is an

2010 Mathematics Subject Classification. Primary: 53C12, 53D05, 58H15. Secondary: 17B70. Keywords: pre-symplectic geometry, deformation theory, foliation, $L_{\infty}$-algebra. 
associated involutive distribution, given by its kernel.

In this note we address equivalences of deformations. For pre-symplectic structures, there is a geometric notion of equivalence: $\eta_{1}$ and $\eta_{2}$ are equivalent if they are related by a diffeomorphism isotopic to the identity. There is also an algebraic notion of equivalence, namely the gauge equivalence of Maurer-Cartan elements of the $L_{\infty}$-algebra $L_{\infty}(M, \eta)$. One of the main results of this note is Thm. 2.17, stating that these two notions essentially coincide when $M$ is compact.

In the same vein, there are also a geometric and an algebraic notion of equivalence for foliations, which we show to coincide in Thm. 2.6. Even though the $L_{\infty}$-algebras controlling the deformations of foliations were investigated already in the early 2000's, to our knowledge the equivalences are addressed here for the first time.

We use these results to draw conclusions about infinitesimal and local properties of the moduli space of pre-symplectic structures (respectively foliations) modulo isotopies. Specifically, we address its smoothness, the dimension of the formal tangent spaces, and whether first order deformations extend to (formal) paths of deformations. We do so in $\$ 2.4$.

The relationship between the equivalences attached to the deformations of pre-symplectic forms and foliations, respectively, is discussed in Remark 2.18.

We finish with a technical note. For both pre-symplectic forms and foliations, the algebraic notion of equivalence is somewhat hard to handle since it is expressed in terms of solutions of a PDE. We bypass this problem by rephrasing algebraic equivalences on $M$ in terms of the product manifold $M \times \mathbb{R}$, see Lemma 2.3 and Lemma 2.12 .

Acknowledgements: M.Z. acknowledges partial support by IAP Dygest, the long term structural funding - Methusalem grant of the Flemish Government, the FWO under EOS project G0H4518N, the FWO research project G083118N (Belgium). We thank the referee for comments that allowed to improve the text, and Stephane Geudens for helpful conversations and useful references.

\section{Review: Deformations of FOLIATIONS AND OF PRE-SyMPlECtiC STRUCTURES}

We recall results on the deformation theory of two kinds of (interrelated) geometric objects: foliations on one side, pre-symplectic forms on the other.

1.1. Deformations. Deformations of a given structure are often controlled by an algebraic structure known as a differential graded Lie algebra or, more generally, an $L_{\infty}$-algebra. An $L_{\infty}$-algebra is a rigorous formalization of the naive notion of a Lie algebra up to homotopy. It consists of the datum of a $\mathbb{Z}$-graded vector space $V$ endowed with degree $(2-k)$ multilinear brackets $\wedge^{k} V \rightarrow V$ satisfying a set of quadratic relations generalizing the Jacobi identity, see [10] for a definition. By shifting degrees by 1 , one gets the equivalent notion of an $L_{\infty}$ [1]-algebra.

Definition 1.1. An $L_{\infty}[1]$-algebra is a $\mathbb{Z}$-graded vector space $W$, equipped with a collection of graded symmetric brackets $\left(\lambda_{k}: W^{\otimes k} \longrightarrow W\right)_{k \geq 1}$ of degree 1 which satisfy the (shifted) higher Jacobi identities of $L_{\infty}$-algebras.

We provide the definition of Maurer-Cartan element only in the case of $L_{\infty}$ [1]-algebras for which all but finitely many multibrackets vanish. For the $L_{\infty}$ [1]-algebras appearing in this note, all the multibrackets vanish except possibly for the first three, namely $\lambda_{1}, \lambda_{2}, \lambda_{3}$.

Definition 1.2. A Maurer-Cartan element of an $L_{\infty}[1]$-algebra $\left(W, \lambda_{1}, \ldots, \lambda_{n}\right)$, where $n \in \mathbb{N}$, is a degree zero element $\beta \in W$ such that

$$
\lambda_{1}(\beta)+\frac{1}{2 !} \lambda_{2}(\beta, \beta)+\cdots+\frac{1}{n !} \lambda_{n}(\beta, \cdots, \beta)=0 .
$$


Given a (algebraic or geometric) object, its deformations are often governed by an $L_{\infty}[1]$ algebra $W$, in the following strong sense: the Maurer-Cartan elements of $W$ are in natural bijection with the "small" deformations of that object. For instance, given an integrable distribution $K$ on a manifold $M$, fix an auxiliary distribution $G$ with $K \oplus G=T M$. In Prop. 1.3 we display an $L_{\infty}$ [1]-algebra whose Maurer-Cartan elements are naturally identified with integrable distributions which are transverse to $G$. We do the same for pre-symplectic forms in Thm. 1.12 , To the best of our knowledge, the first geometric instance of the above phenomenon in which all the multibrackets of the $L_{\infty}$ [1]-algebra can be non-zero, is the work of Oh-Park [15] about deformations of coisotropic submanifolds in symplectic manifolds. For a general discussion of the role of $L_{\infty}$ [1]-algebra in deformation theory, see [4].

\subsection{Foliations and their deformations.}

\subsubsection{Foliations.}

Let $M$ be a smooth manifold. A rank $l$ foliation is a decomposition of $M$ into immersed submanifolds of dimension $l$, subject to a local triviality condition (see for instance [11]).

The celebrated Frobenius theorem states that there is a canonical bijection between foliations on $M$ and involutive distributions on $M$, i.e. subbundles of $T M$ whose sections are stable w.r.t. the Lie bracket of vector fields. More precisely, given a foliation $M$, the associated involutive distribution $D$ is given at any point $p$ as follows: $D_{p}$ is the tangent space to the immersed submanifold (leaf) of the foliation through the point $p$. Because of this bijection, in the following we will work with involutive distributions.

\subsubsection{Deformation theory of foliations.}

We review the $L_{\infty}[1]$-algebra governing deformations of foliations. The following proposition [17, Prop. 4.6] summarizes results by Huebschmann [7], Vitagliano [18] and Xiang Ji [8]:

Proposition 1.3. Let $K$ be an involutive distribution on a manifold $M$, and let $G$ be a complement. There is an $L_{\infty}[1]$-algebra structure on $\Gamma\left(\wedge K^{*} \otimes G\right)[1]$, whose only possibly non-vanishing brackets we denote by $l_{1},-l_{2}, l_{3}$, with the property that the graph of $\phi \in \Gamma\left(K^{*} \otimes G\right)[1]$ is involutive iff $\phi$ is a Maurer-Cartan element.

Rephrasing this proposition, taking the graph of elements of $\Gamma\left(K^{*} \otimes G\right)$ gives a bijection

$$
\mathrm{MC}(K) \rightarrow\{\text { involutive distributions on } M \text { transverse to } G\} \text {, }
$$

where $\mathrm{MC}(K)$ denotes the set of Maurer-Cartan elements of $\left(\Gamma\left(\wedge K^{*} \otimes G\right)[1], l_{1},-l_{2}, l_{3}\right)$.

The formulae for $l_{1}, l_{2}, l_{3}$ are as follows. We remark that $l_{1}$ is the differential associated to the flat $K$-connection on $G$ which, under the identification $G \cong T M / K$, corresponds to the Bott connection. (Thus the underlying cochain complex is the one used by Heitsch [6] to describe infinitesimal deformations of foliations.) For all $\xi \in \Gamma\left(\wedge^{k} K^{*} \otimes G\right)[1], \psi \in \Gamma\left(\wedge^{l} K^{*} \otimes G\right)[1], \phi \in$ $\Gamma\left(\wedge^{m} K^{*} \otimes G\right)[1]$ we have: 


$$
\begin{aligned}
& l_{1}(\xi)\left(X_{1}, \ldots, X_{k+1}\right)=\sum_{i=1}^{k+1}(-1)^{i+1} \operatorname{pr}_{G}\left[X_{i}, \xi\left(X_{1}, \ldots, \widehat{X}_{i}, \ldots, X_{k+1}\right)\right] \\
& +\sum_{i<j}(-1)^{i+j} \xi\left(\left[X_{i}, X_{j}\right], X_{1}, \ldots, \widehat{X}_{i}, \ldots, \widehat{X_{j}}, \ldots, X_{k+1}\right) \\
& l_{2}(\xi, \psi)\left(X_{1}, \ldots, X_{k+l}\right)=(-1)^{k} \sum_{\tau \in S_{k, j}}(-1)^{\tau} \operatorname{pr}_{G}\left[\xi\left(X_{\tau(1)}, \ldots, X_{\tau(k)}\right), \psi\left(X_{\tau(k+1)}, \ldots, X_{\tau(k+l)}\right)\right] \\
& +(-1)^{k(l+1)} \sum_{\tau \in S_{l, 1, k-1}}(-1)^{\tau} \xi\left(\operatorname{pr}_{K}\left[\psi\left(X_{\tau(1)}, \ldots, X_{\tau(l)}\right), X_{\tau(l+1)}\right]\right. \\
& \left., X_{\tau(l+2)}, \ldots, X_{\tau(l+k)}\right) \\
& -(-1)^{k}(\xi \leftrightarrow \psi, k \leftrightarrow l) \\
& l_{3}(\xi, \psi, \phi)\left(X_{1}, \ldots, X_{k+l+m-1}\right)=(-1)^{m+k(l+m)} . \\
& \sum_{\tau \in S_{l, m, k-1}}(-1)^{\tau} \xi\left(\operatorname{pr}_{K}\left[\psi\left(X_{\tau(1)}, \ldots, X_{\tau(l)}\right), \phi\left(X_{\tau(l+1)}, \ldots, X_{\tau(l+m)}\right)\right], \ldots, X_{\tau(l+m+k-1)}\right) \pm \circlearrowleft
\end{aligned}
$$

Here $X_{i} \in \Gamma(K), \operatorname{pr}_{G}$ is the projection $T M=G \oplus K \rightarrow G$, and similarly for $\operatorname{pr}_{K}$. We use $S_{i, j, k}$ to denote the set of permutations $\tau$ of $i+j+k$ elements such that the order is preserved within each block: $\tau(1)<\cdots<\tau(i), \tau(i+1)<\cdots<\tau(i+j), \tau(i+j+1)<\cdots<\tau(i+j+k)$. The symbol $(\xi \leftrightarrow \psi, k \leftrightarrow l)$ denotes the sum just above it, switching $\xi$ with $\psi$ and $k$ with $l$. The symbol $\circlearrowleft$ denotes cyclic permutations in $\xi, \psi, \phi$.

1.3. Pre-symplectic structures and their deformations. After introducing pre-symplectic structures, we review the $L_{\infty}[1]$-algebra governing their deformations, following [17, $\left.\S 1-\S 3\right]$.

\subsubsection{Pre-symplectic structures.}

Definition 1.4. A pre-symplectic structure on a manifold $M$ is an element $\eta \in \Omega^{2}(M)$ such that the rank of $\eta^{\sharp}: T M \rightarrow T^{*} M, v \mapsto \iota_{v} \eta$ is constant, and so that $d \eta=0$.

We define $\operatorname{Pre}^{-S_{y}}{ }^{k}(M):=\{$ pre-symplectic structures of rank $k$ on $M\}$.

Remark 1.5. Let $(M, \eta)$ be a pre-symplectic manifold. Canonically associated to it there is a (constant rank) distribution

$$
K:=\operatorname{ker}\left(\eta^{\sharp}\right),
$$

which is involutive since $d \eta=0$, and thus tangent to a foliation on $M$. We denote the corresponding foliated de Rham complex by $\Omega(K):=\left(\Gamma\left(\wedge K^{*}\right), d_{K}\right)$. It fits in a short exact sequence of cochain complexes

$$
0 \longrightarrow \Omega_{\mathrm{hor}}(M) \longrightarrow \Omega(M) \stackrel{r}{\longrightarrow} \Omega(K) \longrightarrow 0,
$$

where the map $r$ is given by restriction. Notice that its kernel $\Omega_{\text {hor }}(M)$ is generated as a multiplicative ideal of $\Omega(M)$ by $\Gamma\left(K^{\circ}\right)$, where $K^{\circ} \subset T^{*} M$ is the annihilator of $K$. We denote by $H_{\text {hor }}(M)$ the cohomology of $\Omega_{\text {hor }}(M)$.

Remark 1.6. The formal tangent space to $\operatorname{Pre}^{-S_{y m}}{ }^{k}(M)$ at the point $\eta$ is given by

$$
T_{\eta}\left(\operatorname{Pre}_{-S_{y m}}^{k}(M)\right) \cong\left\{\alpha \in \Omega^{2}(M) \text { closed, } r(\alpha)=0\right\}=\left\{2 \text {-cocycles in } \Omega_{\text {hor }}(M)\right) .
$$




\subsubsection{A parametrization inspired by Dirac geometry.}

Fix a finite-dimensional vector space $V$. Any $Z \in \wedge^{2} V$ can be alternatively described by means of the linear map

$$
Z^{\sharp}: V^{*} \rightarrow V, \quad \xi \mapsto \iota_{\xi} Z=Z(\xi, \cdot),
$$

where the antisymmetry of $Z$ is encoded in the following equation for $Z^{\sharp}$ : $\left(Z^{\sharp}\right)^{*}=-Z^{\sharp}$. We define $\mathcal{I}_{Z}$ to consist of all $\beta \in \wedge^{2} V^{*}$ such that id $+Z^{\sharp} \beta^{\sharp}: V \rightarrow V$ is invertible. The formula

$$
(F(\beta))^{\sharp}:=\beta^{\sharp}\left(\mathrm{id}+Z^{\sharp} \beta^{\sharp}\right)^{-1}=\left(\mathrm{id}+\beta^{\sharp} Z^{\sharp}\right)^{-1} \beta^{\sharp}
$$

determines a (non-linear) map $F: \mathcal{I}_{Z} \rightarrow \wedge^{2} V^{*}$, which is a diffeomorphism onto its image $\mathcal{I}_{-Z}$. Denoting by $G$ the image of $Z^{\sharp}$, for any $\beta \in \wedge^{2} V^{*}$ we have: $\beta \in \mathcal{I}_{Z}$ iff $\operatorname{id}_{\mathrm{G}}+Z^{\sharp} \sigma^{\sharp}: G \rightarrow G$ is invertible, where $\sigma:=\left.\beta\right|_{\wedge^{2} G}$.

Now fix $\eta \in \wedge^{2} V^{*}$ of rank $k$. Fix also a subspace $G$ such that $G \oplus K=V$, where $K=\operatorname{ker}\left(\eta^{\sharp}\right)$. Since the restriction $\left.\eta\right|_{G} \in \wedge^{2} G^{*}$ is non-degenerate, there exists a unique $Z \in \wedge^{2} G$ such that $Z^{\sharp}=-\left(\left.\eta\right|_{G} ^{\sharp}\right)^{-1}$.

Definition 1.7. The Dirac exponential map $\exp _{\eta}$ of $\eta$ (and for fixed $G$ ) is the mapping

$$
\exp _{\eta}: \mathcal{I}_{Z} \rightarrow \wedge^{2} V^{*}, \quad \beta \mapsto \eta+F(\beta) .
$$

Let $r: \wedge^{2} V^{*} \rightarrow \wedge^{2} K^{*}$ be the restriction map; we have the natural identification $\operatorname{ker}(r)=$ $\wedge^{2} G^{*} \oplus\left(G^{*} \otimes K^{*}\right)$. The following theorem is [17, Thm. 2.6]. Item (iii) below asserts that, upon restriction to $\operatorname{ker}(r)$, the map $\exp _{\eta}$ is a submanifold chart for $\left(\wedge^{2} V^{*}\right)_{k}$, the space of skewsymmetric bilinear forms on $V$ of rank $k$.

\section{Theorem 1.8.}

(i) Let $\beta \in \mathcal{I}_{Z}$. Then $\exp _{\eta}(\beta)$ lies in $\left(\wedge^{2} V^{*}\right)_{k}$ if, and only if, $\beta$ lies in $\operatorname{ker}(r)=\left(K^{*} \otimes G^{*}\right) \oplus$ $\wedge^{2} G^{*}$.

(ii) Let $\beta=(\mu, \sigma) \in \mathcal{I}_{Z} \cap\left(\left(K^{*} \otimes G^{*}\right) \oplus \wedge^{2} G^{*}\right)$. Then $\exp _{\eta}(\beta)$ is the unique skew-symmetric bilinear form on $V$ with the following properties:

- its restriction to $G$ equals $\left.(\eta+F(\sigma))\right|_{\wedge^{2} G}$

- its kernel is the graph of the map $Z^{\sharp} \mu^{\sharp}=-\left(\left.\eta\right|_{G} ^{\sharp}\right)^{-1} \mu^{\sharp}: K \rightarrow G$.

(iii) The Dirac exponential map $\exp _{\eta}: \mathcal{I}_{Z} \rightarrow \wedge^{2} V^{*}$ restricts to a diffeomorphism

$$
\mathcal{I}_{Z} \cap\left(K^{*} \otimes G^{*}\right) \oplus \wedge^{2} G^{*} \stackrel{\cong}{\longrightarrow}\left\{\eta^{\prime} \in\left(\wedge^{2} V^{*}\right)_{k} \mid \operatorname{ker}\left(\eta^{\prime}\right) \text { is transverse to } G\right\} .
$$

\subsubsection{An $L_{\infty}$-algebra associated to a bivector field.}

Let $Z$ be a bivector field on a manifold $M$. The following results combines [17, Prop. 3.5] and [17. Cor. 3.9] (upon the improvement of the latter obtained in the proof of [16, Cor. 1.9]).

Theorem 1.9. There is an $L_{\infty}[1]$-algebra structure on $\Omega(M)[2]$, whose only possibly nonvanishing multibrackets are $\lambda_{1}, \lambda_{2}, \lambda_{3}$, with the property that for all 2 -forms $\beta \in \Gamma\left(\mathcal{I}_{Z}\right)$ the following statements are equivalent:

(1) $\beta$ is a Maurer-Cartan element of $\left(\Omega(M)[2], \lambda_{1}, \lambda_{2}, \lambda_{3}\right)$,

(2) the 2-form $F(\beta)$ is closed.

Remark 1.10. The differential $\lambda_{1}$ is just the de Rham differential. The binary bracket $\lambda_{2}$ is given (up to signs) by the Koszul bracket associated to $Z$. The trinary bracket $\lambda_{3}$ is obtained by contracting with $\frac{1}{2}[Z, Z]$, in particular it vanishes when $Z$ is a Poisson bivector field. We refer the interested reader to [17] for the details. In this note we do not need the explicit formulae for these multibrackets. 


\subsubsection{The Koszul $L_{\infty}$-algebra of a pre-symplectic manifold.}

Now let $(M, \eta)$ be a pre-symplectic manifold. We fix a subbundle $G$ such that $G \oplus K=T M$, where $K$ is the kernel of $\eta$. Denote by $Z$ the bivector field on $M$ given by $Z^{\sharp}=-\left(\left.\eta\right|_{G} ^{\sharp}\right)^{-1}$. The following is [17, Thm. 3.17].

Theorem 1.11. The $L_{\infty}[1]$-algebra structure on $\Omega(M)[2]$ associated to the bivector field $Z$, see Thm. 1.9, maps $\Omega_{\mathrm{hor}}(M)[2]$ to itself. The subcomplex $\Omega_{\mathrm{hor}}(M)[2] \subset \Omega(M)[2]$ therefore inherits the structure of an $L_{\infty}[1]$-algebra, which we call the $\operatorname{Koszul} L_{\infty}[1]$-algebra of $(M, \eta)$.

From the above one obtains the following main result [17, Thm. 3.19]:

Theorem 1.12. Let $(M, \eta)$ be a pre-symplectic manifold. The choice of a complement $G$ to the kernel of $\eta$ determines a bivector field $Z$ by requiring $Z^{\sharp}=-\left(\left.\eta^{\sharp}\right|_{G}\right)^{-1}$. Suppose $\beta$ is a 2-form on $M$, which lies in $\mathcal{I}_{Z}$. The following statements are equivalent:

(1) $\beta$ is a Maurer-Cartan element of the Koszul $L_{\infty}[1]$-algebra $\Omega_{\mathrm{hor}}(M)[2]$ of $(M, \eta)$,

(2) The image of $\beta$ under the map $\exp _{\eta}$ is a pre-symplectic structure of the same rank as $\eta$.

Here

$$
\exp _{\eta}: \mathcal{I}_{Z} \cap\left(\left(K^{*} \otimes G^{*}\right) \oplus \wedge^{2} G^{*}\right) \rightarrow\left(\wedge^{2} T^{*} M\right)_{k}
$$

is obtained assembling (for every tangent space of $M$ ) the maps introduced in Def. 1.7, followed by restriction. By the above theorem, together with Thm. 1.8, this map induces a bijection

$$
\exp _{\eta}: \Gamma\left(\mathcal{I}_{Z}\right) \cap \mathrm{MC}(\eta) \rightarrow\left\{\eta^{\prime} \in \operatorname{Pre} \operatorname{Sym}^{k}(M) \mid \operatorname{ker}\left(\eta^{\prime}\right) \text { is transverse to } G\right\}
$$

where $\mathrm{MC}(\eta)$ denotes the Maurer-Cartan set of the Koszul $L_{\infty}[1]$-algebra.

1.4. A strict morphism relating deformations. For every pre-symplectic form there is an associated involutive distribution, namely its kernel, and hence by the Frobenius theorem there is also an associated foliation. Following [17, §4], we now show that this can be viewed as the map on Maurer-Cartan elements induced by a strict morphism of $L_{\infty}[1]$-algebras.

Let $(M, \eta)$ be a pre-symplectic manifold, choose a subbundle $G$ such that $G \oplus K=T M$ where $K=\operatorname{ker}\left(\eta^{\sharp}\right)$, and denote by $Z$ the bivector field given by $Z^{\sharp}=-\left(\left.\eta\right|_{G} ^{\sharp}\right)^{-1}$. The following is [17, Prop. 4.6], and states that there is a strict morphism from the $L_{\infty}$ [1]-algebra governing the deformations of the pre-symplectic form $\eta$ to the $L_{\infty}[1]$-algebra governing the deformations of the involutive distribution $K$. Here $F^{2}(\Omega(M)):=\Omega_{\mathrm{hor}}(M) \cdot \Omega_{\mathrm{hor}}(M)$ is an $L_{\infty}$ [1]-ideal of $\Omega_{\text {hor }}(M)[2]$, we denote $\Omega(K, G):=\Gamma\left(\wedge K^{*} \otimes G\right)$, and similarly for $\Omega\left(K, G^{*}\right)$.

Theorem 1.13. The composition

$$
\Omega_{\mathrm{hor}}(M)[2] \rightarrow \Omega_{\mathrm{hor}}(M)[2] / F^{2}(\Omega(M))[2] \cong \Omega\left(K, G^{*}\right)[1] \stackrel{Z^{\sharp}[1]}{\longrightarrow} \Omega(K, G)[1]
$$

is a strict morphism of $L_{\infty}[1]$-algebras, where the domain is the Koszul $L_{\infty}[1]$-algebra with multibrackets $\lambda_{1}, \lambda_{2}, \lambda_{3}$, see Theorem 1.11, and the target $\Omega(K, G)[1]$ is endowed with the multibrackets $l_{1},-l_{2}, l_{3}$, see Prop. 1.3 .

\section{Equivalences}

All the original results of this note are contained in this section. In $₫ 2.2$ we show that the gauge equivalence of foliations essentially agrees with the geometric notion of equivalence given by isotopies, at least in the compact case (see Thm. 2.6). In the long 2.3 we obtain an analog result for pre-symplectic forms (see Thm. 2.17). 
2.1. Gauge equivalence. On the set of Maurer-Cartan elements of an $L_{\infty}$ [1]-algebra there is a canonical equivalence relation. We recall it now for $L_{\infty}[1]$-algebras $W$ for which only the first three multibrackets $\lambda_{1}, \lambda_{2}, \lambda_{3}$ might be non-zero and, as in the two cases of interest for this note, which satisfy the following conditions: $W$ consists of smooth sections of a vector bundle, and each multibracket is a multidifferential operator.

Definition 2.1. Two Maurer-Cartan elements $\beta_{0}$ and $\beta_{1}$ are gauge-equivalent if there is

- a smooth one-parameter family $\left(\beta_{t}\right)_{t \in[0,1]}$ of Maurer-Cartan elements of $W$, agreeing with the given ones at $t=0$ and $t=1$,

- a smooth one-parameter family $\left(\alpha_{t}\right)_{t \in[0,1]}$ of degree -1 elements of $W$,

such that

$$
\frac{\partial}{\partial t} \beta_{t}=\lambda_{1}\left(\alpha_{t}\right)+\lambda_{2}\left(\alpha_{t}, \beta_{t}\right)+\frac{1}{2} \lambda_{3}\left(\alpha_{t}, \beta_{t}, \beta_{t}\right)
$$

As indicated by the name, gauge equivalences define an equivalence relation on the set of Maurer-Cartan elements of $\left(W, \lambda_{1}, \lambda_{2}, \lambda_{3}\right)$. This equivalence relation is also called "homotopy equivalence" in the literature. It can be also phrased as follows: two Maurer-Cartan elements of $W$ are equivalent iff they are of the form $\left.B\right|_{(t=0, d t=0)}$ and $\left.B\right|_{(t=1, d t=0)}$ for some Maurer-Cartan element $B$ of the $L_{\infty}[1]$-algebra $W \otimes \Omega^{\bullet}([0,1])$, where $\Omega^{\bullet}([0,1])$ denotes the cochain complex of differential forms on the unit interval. The differential on $W \otimes \Omega^{\bullet}([0,1])$ is given by the sum of $\lambda_{1}$ and the de Rham differential, while $\lambda_{2}$ and $\lambda_{3}$ are extended to $W \otimes \Omega^{\bullet}([0,1])$ in a $\Omega^{\bullet}([0,1])$-linear fashion with the help of the wedge product on differential forms.

2.2. Equivalences of foliations. Given an involutive distribution on $M$, choose a complement $G$. We saw in Prop. 1.3 that there is an $L_{\infty}$ [1]-algebra whose Maurer-Cartan elements correspond bijectively to involutive distributions transverse to $G$. In this subsection we relate the induced gauge equivalence with the geometric notion of equivalence given by isotopies, see Thm. 2.6 and the text following it.

\subsubsection{Isotopic foliations.}

We introduce the following geometric notion of equivalence for involutive distributions on a manifold $M$.

Definition 2.2. The group $\operatorname{Diff}_{0}(M)$ of diffeomorphisms isotopic to the identity acts on the set of involutive distributions on $M$ from the left, as follows: $f$ maps $D$ to $f_{*}(D)$.

We call two involutive distributions $D$ and $\tilde{D}$ isotopic if they lie in the same orbit of this action.

\subsubsection{Gauge-equivalences of foliations.}

We fix an involutive distribution $K$ on a manifold $M$, and a complement $G$. In Prop. 1.3 we presented an $L_{\infty}[1]$-algebra $\left(\Gamma\left(\wedge K^{*} \otimes G\right)[1], l_{1},-l_{2}, l_{3}\right)$ governing the deformations of the involutive distribution $K$.

By Def. 2.1, two Maurer-Cartan elements $\Phi_{0}, \Phi_{1}$ are gauge equivalent if there is a smooth family $\left(\Phi_{t}\right)_{t \in[0,1]} \subset \Gamma\left(K^{*} \otimes G\right)$ of Maurer-Cartan elements interpolating between them and a smooth family $\left(Y_{t}\right)_{t \in[0,1]}$ in $\Gamma(G)$ such that

$$
\frac{d}{d t} \Phi_{t}=l_{1}\left(Y_{t}\right)-l_{2}\left(Y_{t}, \Phi_{t}\right)+\frac{1}{2} l_{3}\left(Y_{t}, \Phi_{t}, \Phi_{t}\right)
$$

Using the formulae given after Prop. 1.3, one sees that the above equation reads as follows, for all sections $X$ of $K$ :

$$
\frac{d}{d t} \Phi_{t}(X)=-\operatorname{pr}_{G}\left[Y_{t}, X+\Phi_{t}(X)\right]+\Phi_{t}\left(\operatorname{pr}_{K}\left[Y_{t}, X+\Phi_{t}(X)\right]\right)
$$


We give an equivalent characterization of this equation in terms of the product manifold $M \times$ $[0,1]$.

Lemma 2.3. Let $\left(\Phi_{t}\right)_{t \in[0,1]}$ be a smooth one-parameter family in $\Gamma\left(K^{*} \otimes G\right)$ and $\left(Y_{t}\right)_{t \in[0,1]} a$ smooth one-parameter family in $\Gamma(G)$. Denote by $D$ the distribution on $M \times[0,1]$ given by

$$
D_{(p, t)}:=\left\{v+\Phi_{t}(v): v \in K_{p}\right\} \oplus \operatorname{Span}\left(\frac{\partial}{\partial t}+\left.Y_{t}\right|_{p}\right) .
$$

Then $D$ is involutive iff $\Phi_{t}$ consists of Maurer-Cartan elements and the differential equation (5) is satisfied.

Remark 2.4. The distribution $D$ is the graph of the vector bundle map $K \oplus \mathbb{R} \rightarrow G$ (where both are viewed as subbundles of $T(M \times[0,1]))$ which, at points of $M \times\{t\}$, is the sum of $\Phi_{t}$ and of the map $1 \mapsto Y_{t}$.

Proof. Taking sections of $D$ lying in its first or second summand, and using the projection $M \times[0,1] \rightarrow[0,1]$, one sees that the distribution $D$ is involutive iff the following two conditions are satisfied: $\operatorname{graph}\left(\Phi_{t}\right)$ is involutive for all $t$, and for all $X \in \Gamma(K)$ the vector field

$$
\left[X+\Phi_{t}(X), \frac{\partial}{\partial t}+Y_{t}\right]
$$

on $M \times[0,1]$ lies in $D$. Since the vector field (6) projects to zero under $M \times[0,1] \rightarrow[0,1]$, it has no $\frac{\partial}{\partial t}$-component, hence the second condition is equivalent to (6) lying in graph $\left(\Phi_{t}\right)$. This means exactly that the differential equation (5) is satisfied, as one sees writing out (6) as $\left[X+\Phi_{t}(X), Y_{t}\right]-\frac{\partial}{\partial t} \Phi_{t}(X)$ and noticing that the last term lies in $G$.

Proposition 2.5. Let $\left(\Phi_{t}\right)_{t \in[0,1]}$ be any smooth one-parameter family of Maurer-Cartan elements of $\Gamma\left(K^{*} \otimes G\right)$ and $\left(Y_{t}\right)_{t \in[0,1]}$ a one-parameter family in $\Gamma(G)$, such that equation (5) is satisfied. Denote by $\left(\varphi_{t}\right)_{t \in[0,1]}$ the flow of $\left(Y_{t}\right)_{t \in[0,1]}$. We have

$$
\operatorname{graph}\left(\Phi_{t}\right)=\left(\varphi_{t}\right)_{*}\left(\operatorname{graph}\left(\Phi_{0}\right)\right)
$$

at all times $t \in[0,1]$ for which the flow is defined.

Proof. Consider the distribution $D$ appearing in Lemma 2.3 .

We make the following claim: For all $p \in M$ and times $t$ for which $\varphi_{t}$ is defined, we have

$$
D_{(p, t)}=\left(\left(\varphi_{t}\right)_{*} \operatorname{graph}\left(\Phi_{0}\right)\right)_{p} \oplus \operatorname{Span}\left(\frac{\partial}{\partial t}+\left.Y_{t}\right|_{p}\right) .
$$

The claim implies the conclusion, using Remark 2.4

We now prove the claim. The distribution $D$ is involutive, by Lemma 2.3. Consider the vector field $\bar{Y}$ on $M \times \mathbb{R}$ given by $\bar{Y}_{(p, t)}=\frac{\partial}{\partial t}+\left.Y_{t}\right|_{p}$. It is a section of $D$, hence its flow preserves $D$. In particular, the time $t$ flow $F_{t}$ of $\bar{Y}$ satisfies $\left.D\right|_{M \times\{t\}}=\left(F_{t}\right)_{*}\left(\left.D\right|_{M \times\{0\}}\right)$. The r.h.s. agrees with the r.h.s. of eq. (7), as can be computed easily using the fact that the flow $F_{t}$ reads

$$
(p, s) \mapsto\left(\varphi_{t+s}\left(\left(\varphi_{s}\right)^{-1}(p)\right), s+t\right) .
$$

We can now prove the main statement of this subsection:

Theorem 2.6. Let $M$ be a compact manifold with an involutive distribution $K$, and fix a complement $G$. Fix Maurer-Cartan elements $\Phi_{0}, \Phi_{1}$ of $\left(\Gamma\left(\wedge K^{*} \otimes G\right)[1], l_{1},-l_{2}, l_{3}\right)$. The following is equivalent:

(1) $\Phi_{0}$ and $\Phi_{1}$ are gauge equivalent Maurer-Cartan elements of $\left(\Gamma\left(\wedge K^{*} \otimes G\right)[1], l_{1},-l_{2}, l_{3}\right)$ 
(2) There is a diffeomorphism $\varphi$ of $M$ such that

$$
\varphi_{*}\left(\operatorname{graph}\left(\Phi_{0}\right)\right)=\operatorname{graph}\left(\Phi_{1}\right),
$$

and so that $\varphi$ is connected to the identity by an isotopy $\left(\varphi_{t}\right)_{t \in[0,1]}$ with the property that, for all $t \in[0,1],\left(\varphi_{t}\right)_{*}\left(\operatorname{graph}\left(\Phi_{0}\right)\right)$ is transverse to $G$.

Thm. 2.6 shows that two involutive distributions transverse to $G$ are gauge equivalent iff they lie in the same connected component of

$$
\{\text { involutive distributions transverse to } G\} \cap\left(\operatorname{aiff}_{0}(M)\right. \text {-orbit). }
$$

Proof. "(1) $\Rightarrow(2)$ ": apply Prop. 2.5, noticing that the compactness of $M$ assures that all flows are defined on $[0,1]$.

"(2) $\Rightarrow(1)$ ". Consider the diffeomorphism $\bar{\varphi}$ of $M \times[0,1]$ given by

$$
(p, t) \mapsto\left(\varphi_{t}(p), t\right) .
$$

Consider the distribution on $M \times[0,1]$ given by $\operatorname{graph}\left(\Phi_{0}\right) \oplus \operatorname{Span}\left(\frac{\partial}{\partial t}\right)$. It is an involutive distribution, being the product of two involutive ones. One computes that its push-forward by $\bar{\varphi}$ is the distribution which at each point $(p, t) \in M \times[0,1]$ reads

$$
\left(\varphi_{t}\right)_{*}\left(\operatorname{graph}\left(\Phi_{0}\right)\right) \oplus \operatorname{Span}\left(\frac{\partial}{\partial t}+\left.V_{t}\right|_{p}\right)
$$

where ${ }^{1} V_{t}:=\left(\frac{d}{d t} \varphi_{t}\right)$. This distribution is exactly the distribution $D$ appearing in Lemma 2.3 associated to the data $\left(\Phi_{t}, Y_{t}\right)_{t \in[0,1]}$, where

$\Phi_{t} \in \Gamma\left(K^{*} \otimes G\right)$ is defined by the requirement that $\operatorname{graph}\left(\Phi_{t}\right)=\left(\varphi_{t}\right)_{*}\left(\operatorname{graph}\left(\Phi_{0}\right)\right)$,

$Y_{t} \in \Gamma(G)$ is the image of $V_{t}$ under the projection $T M \rightarrow G$ with kernel $\left(\varphi_{t}\right)_{*}\left(\operatorname{graph}\left(\Phi_{0}\right)\right)$. Hence $D$ is involutive. Using Lemma 2.3 we obtain (1).

Remark 2.7. [On isotopies tangent to $G$ ] Given a diffeomorphism $\varphi$ as in Thm. 2.6 (2), one can constrict a diffeomorphism $\varphi^{\prime}$ with the same properties and so that the isotopy $\left(\varphi_{t}^{\prime}\right)_{t \in[0,1]}$ in addition satisfies $\left(\frac{d}{d t} \varphi_{t}^{\prime}\right) \in \Gamma(G)$. This geometric fact follows from the proof of the implication " $(1) \Rightarrow(2)$ " in the above theorem.

Remark 2.8. An involutive distribution $K$ on a manifold $M$ is a Lie subalgebroid of $T M$, hence the vector bundle $K$ acquires a Lie algebroid structure with injective anchor map. Consider the DGLA of Crainic-Moerdijk [3] governing deformations of this Lie algebroid structure. Its gaugeequivalences were determined by La Pastina-Vitagliano [9, §1] when $M$ is compact, and read as follows: two Lie algebroid structures on $K$ with injective anchor are equivalent iff the images of their anchor maps are isotopic. The DGLA of Crainic-Moerdijk is $L_{\infty}$-quasi-isomorphic to the $L_{\infty}$-algebra of Prop. 1.3 [18, App. C]. Following a suggestion by Luca Vitagliano, one might try to show that the gauge-equivalence classes of Maurer-Cartan elements of the former DGLA are in bijection with those of the latter $L_{\infty}$-algebra, thus giving another approach to the deformation problem of foliations modulo isotopy. To do so, a necessary step is to check that the $L_{\infty}$-quasi-isomorphism relating them is sufficiently well-behaved as not to give rise to any convergence problems.

2.3. Equivalences of pre-symplectic structures. Given a pre-symplectic form $\eta$ on $M$ and a choice a complement to its kernel, we saw in $\$ 1.3 .4$ that there is an $L_{\infty}[1]$-algebra structure on $\Omega_{\text {hor }}(M)[2]$ whose Maurer-Cartan elements parametrize pre-symplectic forms nearby $\eta$, via the Dirac exponential map $\beta \mapsto \exp _{\eta}(\beta)$ of Def. 1.7. In this subsection we relate the gauge equivalence of Maurer-Cartan elements with the geometric notion of isotopic pre-symplectic forms, see Thm. 2.17 and the text following it.

\footnotetext{
${ }^{1}$ Explicitly, $V_{t}(p)=\left.\frac{d}{d s}\right|_{s=t}\left(\varphi_{s}\left(\varphi_{t}^{-1}(p)\right)\right)$.
} 
2.3.1. Isotopic pre-symplectic structures. We start introducing a notion of equivalence of presymplectic structures on a manifold $M$, which is natural from the geometric point of view.

Definition 2.9. The group $\operatorname{Diff}_{0}(M)$ of diffeomorphisms isotopic to the identity acts on $\operatorname{Pre}^{-S_{y m}}{ }^{k}(M)$ from the right via $\eta \cdot f:=f^{*} \eta$.

We call two pre-symplectic structures $\eta$ and $\tilde{\eta}$ isotopic if they lie in the same orbit of this action, and then write $\eta \sim \tilde{\eta}$.

Furthermore, we denote the set of orbits by $\operatorname{Pre-Sym}^{k}(M) / \operatorname{Diff}_{0}(M)$.

We will need a reformulation of the equivalence relation $\sim:$

Proposition 2.10. Suppose $M$ is compact. Two pre-symplectic structures $\eta$ and $\tilde{\eta}$ on $M$ are isotopic, if and only if there is a smooth one-parameter family of pre-symplectic structures $\left(\eta_{t}\right)_{t \in[0,1]}$ joining them, such that the variation $\frac{d}{d t} \eta_{t}$ equals $d \gamma_{t}$, with $\gamma_{t}$ a section of $\left(\operatorname{ker}\left(\eta_{t}\right)\right)^{\circ}$ depending smoothly on $t$.

Above, $\left(\operatorname{ker}\left(\eta_{t}\right)\right)^{\circ} \subset T^{*} M$ is the annihilator of $\operatorname{ker}\left(\eta_{t}\right) \subset T M$.

Proof. Assume that $\eta$ and $\tilde{\eta}$ are isotopic via $\left(f_{t}\right)_{t \in[0,1]}$, in particular $f_{1}^{*} \eta=\tilde{\eta}$. Then the smooth one-parameter family $\eta_{t}:=\left(f_{t}\right)^{*} \eta$ satisfies the requirements of the proposition since

$$
\frac{d}{d t} \eta_{t}=\left(f_{t}\right)^{*}\left(\mathcal{L}_{X_{t}} \eta\right)=d \iota_{\left(f_{t}^{-1}\right)_{*} X_{t}} \eta_{t},
$$

and $\iota_{\left(f_{t}^{-1}\right)_{*} X_{t}} \eta_{t}$ lies in $\left(\operatorname{ker}\left(\eta_{t}\right)\right)^{\circ}$. Here $X_{t}$ is the time-dependent vector field associated to the isotopy.

One the other hand, if we are given a families $\left(\eta_{t}\right)_{t \in[0,1]}$ and $\left(\gamma_{t}\right)_{t \in[0,1]}$ as specified in the proposition, we can apply Moser's trick. In more detail, we make the Ansatz

$$
0=\frac{d}{d t}\left(g_{t}^{*} \eta_{t}\right)=g_{t}^{*}\left(d \iota_{X_{t}} \eta_{t}+\frac{d}{d t} \eta_{t}\right)=g_{t}^{*} d\left(\iota_{X_{t}} \eta_{t}+\gamma_{t}\right)
$$

for the isotopy $\left(g_{t}\right)_{t \in[0,1]}$ generated by $X_{t}$. Now we can find a one-parameter family of vector fields $X_{t}$ such that

$$
\iota_{X} \eta_{t}+\gamma_{t}=0
$$

since $\gamma_{t}$ lies in the image of $\eta_{t}^{\sharp}$. Observe that the kernels of $\eta_{t}$, as $t$ ranges over [0,1], form a vector bundle over $M \times[0,1]$ and we can choose a complementary subbundle to it inside the pull-back of $T M$. Requiring that $X_{t}$ takes values in this subbundle uniquely determines the one-parameter family $X_{t}$ (this shows in particular that $X_{t}$ can be chosen in a smooth manner). Since $M$ is compact, $\left(X_{t}\right)_{t \in[0,1]}$ will integrate to an isotopy $\left(g_{t}\right)_{t \in[0,1]}$. Setting $f_{t}:=g_{t}^{-1}$ yields the desired isotopy satisfying $\eta_{t}=f_{t}^{*} \eta$, in particular $\tilde{\eta}=f_{1}^{*} \eta$.

Remark 2.11. Let us determine the formal tangent space to $\operatorname{Pre-Sym}{ }^{k}(M) / \operatorname{Diff}_{0}(M)$ at the equivalence class of $\eta$. By Remark 1.6, the formal tangent space of $\operatorname{Pre}^{-S_{y m}}{ }^{k}(M)$ at $\eta$ can be identified with the closed 2-forms on $M$ whose restriction to $K=\operatorname{ker}(\eta)$ is zero. On the other hand, by Prop. 2.10, the equivalence class of $\eta$ is infinitesimally modelled by $d \beta$ for $\beta \in \Gamma\left(K^{\circ}\right)$. As the quotient of these two vector spaces, and hence as the candidate for $T_{[\eta]}\left(\operatorname{Pre}_{-} \operatorname{Sym}^{k}(M) / \operatorname{Diff}_{0}(M)\right)$, we therefore find $H_{\text {hor }}^{2}(M)$.

\subsubsection{Gauge-equivalences of two-forms.}

Fix a bivector field $Z$ on $M$. Recall that associated to $Z$, we constructed an $L_{\infty}[1]$-algebra structure on $\Omega(M)[2]$ with structure maps $\lambda_{1}, \lambda_{2}$ and $\lambda_{3}$, in Thm. 1.9. Here we give a characterization of when two sufficiently small Maurer-Cartan elements are gauge equivalent in terms of the map $F$ introduced in eq. (2), see Prop. 2.14 below. This will be used to prove Thm. 2.17 , which gives a geometric characterization of when two sufficiently small Maurer-Cartan elements 
of $\Omega_{\text {hor }}(M)[2]$ are gauge equivalent. In the latter statement, "sufficiently small" means that the two Maurer-Cartan elements take value in the neighborhood $\mathcal{I}_{Z}$ of the zero section of $\wedge^{2} T^{*} M$ introduced before eq. (2), and can be joined by a smooth curve of Maurer-Cartan elements taking values in $\mathcal{I}_{Z}$.

For given Maurer-Cartan elements $\beta_{0}, \beta_{1} \in \Omega^{2}(M)[2]$, we defined gauge equivalence in Def. 2.1. requiring the existence of smooth one-parameter families $\left(\beta_{t}\right)_{t \in[0,1]} \subset \Omega^{2}(M)[2]$ of MaurerCartan elements and $\left(\alpha_{t}\right)_{t \in[0,1]} \subset \Omega^{1}(M)[2]$ satisfying eq. (4). We characterize this in terms of the product manifold $M \times[0,1]$, just as we did in Lemma 2.3 .

Lemma 2.12. Two Maurer-Cartan elements $\beta_{0}$ and $\beta_{1}$ are gauge equivalent iff there is

- a smooth one-parameter family $\left(\beta_{t}\right)_{t \in[0,1]}$ in $\Omega^{2}(M)[2]$ joining $\beta_{0}$ to $\beta_{1}$

- a smooth one-parameter family $\left(\alpha_{t}\right)_{t \in[0,1]}$ in $\Omega^{1}(M)[2]$

such that

$$
\beta_{t}+d t \wedge \alpha_{t} \in \Omega^{2}(M \times[0,1])[2]
$$

is a Maurer-Cartan element in the $L_{\infty}[1]$-algebra

$$
\left(\Omega(M \times[0,1])[2], \lambda_{1}+d_{[0,1]}, \lambda_{2}, \lambda_{3}\right) .
$$

Here the operator $d_{[0,1]}$ is the de Rham differential on the interval $[0,1]$, while $\lambda_{i}$ denotes the $\Omega([0,1])$-linear extensions of $\lambda_{i}$ from $\Omega(M)$ to $\Omega(M \times[0,1])$, and the Koszul sign convention is understood.

Proof. By a straightforward computation, $\beta_{t}+d t \wedge \alpha_{t}$ is a Maurer-Cartan element iff $\left(\beta_{t}\right)_{t \in[0,1]}$ and $\left(\alpha_{t}\right)_{t \in[0,1]}$ satisfy eq. (4).

Consider the manifold $M \times[0,1]$, equipped with the bivector field $\widetilde{Z}$ defined by $\widetilde{Z}_{(p, t)}=$ $\left(Z_{p}, 0\right) \in T_{(p, t)}(M \times[0,1])$. Denote by $\widetilde{F}: \mathcal{I}_{\widetilde{Z}} \rightarrow \mathcal{I}_{-\widetilde{Z}}$ the bijection $\gamma \mapsto \gamma^{\sharp}\left(\mathrm{id}+\widetilde{Z}^{\sharp} \gamma^{\sharp}\right)^{-1}$ between neighborhoods of the origin in $\Omega^{2}(M \times[0,1])$, as in eq. (2). The $L_{\infty}$ [1]-algebra structure associated to $\widetilde{Z}$ by Thm. 1.9 is exactly $\left(\Omega(M \times[0,1])[2], \lambda_{1}+d_{[0,1]}, \lambda_{2}, \lambda_{3}\right)$ as introduced in Lemma 2.12. Given a Maurer-Cartan element $\beta_{t}+d t \wedge \alpha_{t} \in \Omega(M \times[0,1])[2]$ lying in $\mathcal{I}_{\widetilde{Z}}$, we know by Thm. 1.9 that the image

$$
\widetilde{F}\left(\beta_{t}+d t \wedge \alpha_{t}\right)=: \widehat{\beta}_{t}+d t \wedge \widehat{\alpha}_{t}
$$

is closed with respect to the de Rham differential, which in turn amounts to

$$
d \widehat{\beta}_{t}=0 \quad \text { and } \quad \frac{\partial \widehat{\beta}_{t}}{\partial t}=d \widehat{\alpha}_{t} .
$$

Notice that $\beta_{t}+d t \wedge \alpha_{t} \in \Omega(M \times[0,1])$ lies in $\mathcal{I}_{\widetilde{Z}}$ iff $\beta_{t}$ lies in $\mathcal{I}_{Z}$ for all $t$, see $\$ 1.3 .2$. Since $\widetilde{F}$ maps $\mathcal{I}_{\widetilde{Z}}$ bijectively onto $\mathcal{I}_{-\widetilde{Z}}$, it follows that $\widehat{\beta}_{t}$ lies in $\mathcal{I}_{-Z}$ for all $t$.

We compute explicitly the r.h.s. of eq. (8).

Lemma 2.13. For a family $\alpha_{t}$ of 1 -forms on $M$ and a family $\beta_{t}$ of 2 -forms lying in $\mathcal{I}_{Z}$ we have

$$
\widetilde{F}\left(\beta_{t}+d t \wedge \alpha_{t}\right)=F\left(\beta_{t}\right)+d t \wedge F^{\prime}\left(\beta_{t}, \alpha_{t}\right) \quad \in \Omega^{2}(M \times[0,1]) .
$$

Here for any pair $(\beta, \alpha) \in \Gamma\left(\mathcal{I}_{Z}\right) \times \Omega^{1}(M)$ we denote

$$
\widehat{\beta}^{\sharp}:=F(\beta)^{\sharp}=\beta^{\sharp}\left(\mathrm{id}+Z^{\sharp} \beta^{\sharp}\right)^{-1} \text { as in eq. (2), } \quad \widehat{\alpha}:=F^{\prime}(\beta, \alpha):=\left(\mathrm{id}+\beta^{\sharp} Z^{\sharp}\right)^{-1} \alpha \text {. }
$$

\footnotetext{
${ }^{2}$ Explicitly, at a point $(p, t) \in M \times[0,1]$ the above 2 -form reads $\left(\beta_{t}\right)_{p}+d t \wedge\left(\alpha_{t}\right)_{p}$.
} 
Proof. We have

$$
\widetilde{F}\left(\beta_{t}+d t \wedge \alpha_{t}\right)^{\sharp}=\left[\mathrm{id}+\left(\beta_{t}+d t \wedge \alpha_{t}\right)^{\sharp} \widetilde{Z}^{\sharp}\right]^{-1}\left(\beta_{t}+d t \wedge \alpha_{t}\right)^{\sharp} .
$$

W.r.t. the splitting $T_{(p, t)}^{*}(M \times[0,1])=T_{p}^{*} M \oplus \mathbb{R} d t$, the isomorphism in the square bracket is lower triangular, so it can be easily inverted to obtain $\left(\begin{array}{cc}\left(\mathrm{id}+\beta^{\sharp} Z^{\sharp}\right)^{-1} & 0 \\ d t \cdot \alpha^{\sharp} Z^{\sharp}\left(\mathrm{id}+\beta^{\sharp} Z^{\sharp}\right)^{-1} & \mathrm{id}_{\mathbb{R} d t}\end{array}\right)$. Using this, it is straightforward to verify that the above 2 -form on $M \times[0,1]$ simplifies to $F\left(\beta_{t}\right)+d t \wedge F^{\prime}\left(\beta_{t}, \alpha_{t}\right)$ as stated in the proposition.

In conclusion, we obtain:

Proposition 2.14. Fix a bivector field $Z$ on $M$. There is a bijection between:

(1) One-parameter families $\left(\beta_{t}\right)_{t \in[0,1]} \subset \mathcal{I}_{Z}$ of Maurer-Cartan elements of $\left(\Omega(M)[2], \lambda_{1}, \lambda_{2}, \lambda_{3}\right)$, one-parameter families $\left(\alpha_{t}\right)_{t \in[0,1]}$ in $\Omega^{1}(M)$ such that the differential equation (4) is satisfied,

(2) One-parameter families $\left(\widehat{\beta}_{t}\right)_{t \in[0,1]} \subset \mathcal{I}_{-Z}$ of closed forms in $\Omega^{2}(M)$ and one-parameter families $\left(\widehat{\alpha}_{t}\right)_{t \in[0,1]}$ in $\Omega^{1}(M)$ satisfying $\frac{\partial \widehat{\beta}_{t}}{\partial t}=d \widehat{\alpha}_{t}$.

The bijection maps $\left(\beta_{t}, \alpha_{t}\right)_{t \in[0,1]}$ to $\left(\widehat{\beta}_{t}, \widehat{\alpha}_{t}\right)_{t \in[0,1]}$ as in eq. (10).

Proof. Given an element of the set (1), apply Lemma 2.12 (and its proof) and the map $\widetilde{F}$ to obtain an element of the set (2). This map is a bijection since $\widetilde{F}: \mathcal{I}_{\widetilde{Z}} \rightarrow \mathcal{I}_{-\widetilde{Z}}$ is a bijection. The formulae for $\widehat{\beta}_{t}$ and $\widehat{\alpha}_{t}$ were obtained in Lemma 2.13 .

\subsubsection{Gauge equivalences of pre-symplectic forms.}

Let $(M, \eta)$ be a pre-symplectic manifold. As usual, we fix a complement $G$ of the kernel $K=\operatorname{ker}(\eta)$ and denote by $Z$ the bivector field determined by $Z^{\sharp}=-\left(\left.\eta\right|_{G} ^{\sharp}\right)^{-1}$.

The subcomplex of $\left(\Omega(M)[2], \lambda_{1}, \lambda_{2}, \lambda_{3}\right)$ consisting of horizontal differential forms $\Omega_{\mathrm{hor}}(M)$ forms an $L_{\infty}$ [1]-subalgebra, which we refer to as the Koszul $L_{\infty}[1]$-algebra of $(M, \eta)$, see Thm. 1.11. Further we saw that the Maurer-Cartan elements of the Koszul $L_{\infty}$ [1]-algebra correspond to deformations of the pre-symplectic structure $\eta$ (the rank is required to stay fixed), see Thm. 1.12. Now we specialize to horizontal forms the results about gauge equivalence obtained in \$2.3.2.

We first need a linear algebra lemma. Recall that $\widehat{\alpha}, \widehat{\beta}$ were defined in eq. 10 .

Lemma 2.15. Let $\beta \in \Omega_{\text {hor }}^{2}(M)$ lie in $\mathcal{I}_{Z}$. For any $\alpha \in \Omega^{1}(M)$ we have: $\alpha \in \Omega_{\text {hor }}^{1}(M)$ iff $\widehat{\alpha}$ vanishes on the kernel of $\eta+\widehat{\beta}$.

Proof. The kernel of $\eta+\widehat{\beta}$ equals the image of the map (id $\left.+\left.Z^{\sharp} \beta^{\sharp}\right|_{K}\right): K \rightarrow T M$, by Theorem 1.8. For all $v \in K$ we have

$$
\left\langle\widehat{\alpha},\left(\mathrm{id}+Z^{\sharp} \beta^{\sharp}\right)(v)\right\rangle=\left\langle\left(\mathrm{id}+\beta^{\sharp} Z^{\sharp}\right)^{-1}(\alpha),\left(\mathrm{id}+Z^{\sharp} \beta^{\sharp}\right)(v)\right\rangle=\langle\alpha, v\rangle,
$$

proving the lemma.

We can now refine Prop. 2.14 to:

Proposition 2.16. There is a bijection between:

(1) One-parameter families $\left(\beta_{t}\right)_{t \in[0,1]} \subset \mathcal{I}_{Z}$ of Maurer-Cartan elements of $\left(\Omega_{\mathrm{hor}}(M)[2], \lambda_{1}, \lambda_{2}, \lambda_{3}\right)$, one-parameter families $\left(\alpha_{t}\right)_{t \in[0,1]}$ in $\Omega_{\text {hor }}^{1}(M)$ such that the differential equation (4) is satisfied. 
(2) One-parameter families $\left(\widehat{\beta}_{t}\right)_{t \in[0,1]}$ in $\Omega^{2}(M)$ and one-parameter families $\left(\widehat{\alpha}_{t}\right)_{t \in[0,1]}$ in $\Omega^{1}(M)$

such that

- $\eta_{t}:=\eta+\widehat{\beta}_{t}$ is pre-symplectic on $M$ of rank equal to rank( $\left.\eta\right)$ and with kernel transverse to $G$,

- $\widehat{\alpha}_{t}$ vanishes on $\operatorname{ker}\left(\eta_{t}\right)$,

- $\frac{\partial \eta_{t}}{\partial t}=d \widehat{\alpha_{t}}$.

Proof. Use Prop. 2.14, together with Thm. 1.12 with the text following it, and Lemma 2.15.

Rephrasing the above proposition gives the main result of this subsection.

Theorem 2.17. Let $(M, \eta)$ be a compact pre-symplectic manifold, and fix a complement $G$ of $\operatorname{ker}(\eta)$. Fix Maurer-Cartan elements $\beta_{i}$ of $\left(\Omega_{\mathrm{hor}}(M)[2], \lambda_{1}, \lambda_{2}, \lambda_{3}\right)$ lying in $\mathcal{I}_{Z}$, and denote by $\eta_{i}:=\eta+F\left(\beta_{i}\right)$ the corresponding pre-symplectic forms, for $i=0,1$. The following is equivalent:

(1) The Maurer-Cartan elements $\beta_{0}$ and $\beta_{1}$ are gauge equivalent through Maurer-Cartan elements $\left(\beta_{t}\right)_{t \in[0,1]}$ lying in $\mathcal{I}_{Z}$.

(2) There is a diffeomorphism of $M$ pulling back $\eta_{1}$ to $\eta_{0}$ and isotopic to the identity by an isotopy $\left(\varphi_{t}\right)_{t \in[0,1]}$ such that $\left(\varphi_{t}\right)_{*}\left(\operatorname{ker}\left(\eta_{0}\right)\right)$ is transverse to $G$ for all $t$.

Proof. Apply Prop. 2.16 and Prop. 2.10.

Thm. 2.17 shows that two Maurer-Cartan elements lying in $\mathcal{I}_{Z}$ are gauge equivalent through Maurer-Cartan elements lying in $\mathcal{I}_{Z}$ iff they lie in the same connected component of

$\{$ pre-symplectic forms with kernel transverse to $G\} \cap$ (a $\operatorname{Diff}_{0}(M)$-orbit).

Remark 2.18. [On isotopies tangent to $G$, and relation to Thm. 2.6

Suppose we are given $\beta_{0}, \beta_{1} \in \Omega_{\text {hor }}^{2}(M)$ as in the first item of Thm. 2.17, that is: they are gauge-equivalent via one-parameter families $\left(\alpha_{t}, \beta_{t}\right)_{t \in[0,1]}$ with $\beta_{t} \in \mathcal{I}_{Z}$.

(i) An isotopy as in the second item of Thm. 2.17 is obtained by integrating $Z^{\sharp} \alpha_{t}$. Indeed, the isotopy constructed in Prop. 2.10 is obtained by integrating a time-dependent vector field $X_{t}$ satisfying $\iota_{X} \eta_{t}=-\widehat{\alpha}_{t}$. We claim that $X_{t}:=Z^{\sharp} \alpha_{t}$ (a vector field tangent to the distribution $G)$ satisfies this equation. The claim follows from

$$
\iota_{Z^{\sharp} \alpha_{t}} \eta_{t}=-\alpha_{t}+\beta_{t}^{\sharp}\left(i d+Z^{\sharp} \beta_{t}^{\sharp}\right)^{-1} Z^{\sharp} \alpha_{t}=-\left(i d+\beta_{t}^{\sharp} Z^{\sharp}\right)^{-1} \alpha_{t}=-\widehat{\alpha}_{t},
$$

where we used $\beta_{t}^{\sharp}\left(i d+Z^{\sharp} \beta_{t}^{\sharp}\right)^{-1} Z^{\sharp}=i d-\left(i d+\beta_{t}^{\sharp} Z^{\sharp}\right)^{-1}$ in the second equality.

This implies the following geometric fact, analogous to Lemma 2.7 given a diffeomorphism $\varphi$ as in Thm. 2.17 (2), one can construct a diffeomorphism $\varphi^{\prime}$ with the same properties and so that in addition the isotopy $\left(\varphi_{t}^{\prime}\right)_{t \in[0,1]}$ satisfies $\left(\frac{d}{d t} \varphi_{t}^{\prime}\right) \in \Gamma(G)$.

(ii) In Thm. 1.13 we displayed a strict $L_{\infty}[1]$-morphism from $\Omega_{\text {hor }}(M)[2]$ to $\Gamma\left(\wedge K^{*} \otimes G\right)[1]$, the $L_{\infty}$ [1]-algebra which governs deformations of involutive distributions transverse to $G$. Applying this morphism to $\beta_{i}(i=0,1)$ we obtain Maurer-Cartan elements $\Phi_{i}:=\left.Z^{\sharp} \beta_{i}\right|_{K}$ of $\Gamma\left(\wedge K^{*} \otimes G\right)[1]$ which are gauge-equivalent, via $\left(Z^{\sharp} \alpha_{t}, Z^{\sharp} \beta_{t}\right)_{t \in[0,1]}$. Notice that graph $\left(\Phi_{i}\right)=\operatorname{ker}\left(\eta_{i}\right)$ by Thm. 1.8

On the other hand, in view of (i) above, the isotopy $\left(\varphi_{t}\right)_{t \in[0,1]}$ obtained integrating $Z^{\sharp} \alpha_{t}$ satisfies the properties in Thm. $2.17(2)$. In particular, $\left(\varphi_{1}\right)_{*}\left(\operatorname{ker}\left(\eta_{0}\right)\right)=\operatorname{ker}\left(\eta_{1}\right)$ and $\left(\varphi_{t}\right)_{*}\left(\operatorname{ker}\left(\eta_{0}\right)\right)$ is transverse to $G$ for all $t \in[0,1]$.

The two results explained in this item are consistent with Thm. 2.6 on equivalences of foliations.

Remark 2.19. [An alternative proof of Prop. 2.16 Our Prop. 2.16 was proven specializing Prop. 2.14 to horizontal forms on $M$, by means of Lemma 2.15 
We provide an alternative proof of Prop. 2.16, working on the product $\widetilde{M}:=M \times[0,1]$ and specializing Lemma 2.13 to horizontal forms on $M$. We view $\eta$ as a 2 -form on $\widetilde{M}$, by taking its pullback. It is pre-symplectic, with kernel $K \oplus \mathbb{R} \partial_{t}$. Notice that a complement to this kernel in $T \widetilde{M}$ is (the obvious extension of) $G$.

Take one-parameter families $\left(\beta_{t}\right)_{t \in[0,1]}$ and $\left(\alpha_{t}\right)_{t \in[0,1]}$ as in (1) of Prop. 2.16. The 2-form $\beta_{t}+d t \wedge \alpha_{t}$ on $\widetilde{M}$ is clearly horizontal, and is a Maurer-Cartan element by Lemma 2.12. Hence by applying $\widetilde{F}$ as in eq. (8) we see (using Thm. 1.8 and Thm. 1.12 that

$$
\left(\eta+\widehat{\beta}_{t}\right)+d t \wedge \widehat{\alpha}_{t}
$$

is a pre-symplectic form on $\widetilde{M}$ whose kernel has dimension $\operatorname{dim}(K)+1$ and is transverse to $G$.

Notice that, for all $t$, the condition $\widehat{\alpha}_{t} \in\left(\operatorname{ker}\left(\eta+\widehat{\beta}_{t}\right)\right)^{\circ}$ holds at every point of $M$. (This follows from eq. (12), using that $\operatorname{dim}\left(\operatorname{ker}\left(\eta+\widehat{\beta}_{t}\right)\right)=\operatorname{dim}(K)$ by Thm. 1.8 (i).) Applying Cor. A.2 and using eq. (11) we obtain item (2) in Prop. 2.16. Clearly this reasoning can be inverted.

2.4. Moduli spaces. We collect some geometric corollaries about the space of foliations (respectively pre-symplectic structures) modulo isotopy.

2.4.1. Moduli space of foliations. Denote by $\mathrm{Fol}^{k}(M)$ the space of codimension $k$ involutive distributions on a manifold $M$. Recall that $\mathrm{Fol}^{k}(M)$ carries an action of the group of isotopies $\operatorname{Diff}_{0}(M)$ (Def. 2.2).

Fix an involutive distribution $K$. Thanks to Prop. 1.3 and Thm. 2.6, we know that the formal tangent space to the moduli space $\mathrm{Fol}^{k}(M) / \operatorname{Diff}_{0}(M)$ at the equivalence class of $K$ is

$$
\left.T_{[K]}\left(\operatorname{Fol}^{k}(M)\right) / \operatorname{Diff}_{0}(M)\right) \cong H^{1}(K, G) .
$$

Here $G$ is an auxiliary distribution complementary to $K$, and $H(K, G)$ denotes the cohomology of the complex $\left(\Gamma\left(\wedge K^{*} \otimes G\right), l_{1}\right)$ introduced just after Prop. 1.3. We now show that the dimension of this tangent space can vary abruptly.

Lemma 2.20. Let $M=\mathbb{T}^{2}$ be the 2-torus with "coordinates" $\theta_{1}$ and $\theta_{2}$. For any $\lambda \in \mathbb{R}$ consider the involutive distribution $K_{\lambda}:=\operatorname{Span}\left\{\frac{\partial}{\partial \theta_{1}}+\lambda \frac{\partial}{\partial \theta_{2}}\right\}$, and its complement $G=\operatorname{Span}\left\{\frac{\partial}{\partial \theta_{2}}\right\}$. Then

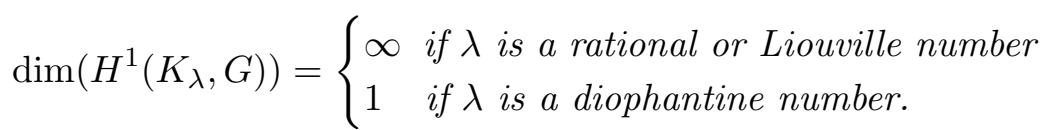

Here we call an irrational number $\lambda$ diophantine if there exists a positive real number $s$ such that $\inf \left\{|m \lambda+n|\left(1+m^{2}\right)^{s}\right\}>0$, where the infimum ranges over all $(m, n) \in \mathbb{Z}^{2} \backslash\{0\}$. The Liouville numbers are the irrational numbers that are not diophantine; they have the property that they can be well approximated by rational numbers.

Proof. By means of the line bundle isomorphism $G \cong \mathbb{R} \times M$ mapping the section $\frac{\partial}{\partial \theta_{2}}$ to the constant section 1, the Bott connection on $G$ is identified with the foliated de Rham differential acting on functions. This means that the differential $l_{1}$ can be identified with the foliated de Rham differential, and therefore $H\left(K_{\lambda}, G\right)$ is isomorphic to the foliated cohomology $H\left(K_{\lambda}\right)$.

If $\lambda$ is rational, then the foliation $K_{\lambda}$ is the fiber foliation of a principal $U(1)$-bundle $M \rightarrow$ $S^{1}$, and since the fibers have non-trivial first cohomology it follows that $H^{1}\left(K_{\lambda}\right)$ is infinitedimensional. If $\lambda$ is a Liouville or diophantine number, for a computation of $H^{1}\left(K_{\lambda}\right)$ using Fourier analysis we refer to [5, §2.1] (see also [12, page 60]).

Remark 2.21 (Obstructedness). In general, the deformation problem of foliations is formally obstructed. This means that in general there is a closed element of $\Gamma\left(K^{*} \otimes G\right)$ - in other words, a first order deformation - that can not be extended to a formal curve of Maurer-Cartan 
elements. This was shown in [17, $\S 6.2]$, by taking the underlying manifold to be the 4 -torus $\mathbb{T}^{4}$ and $K=\operatorname{Span}\left\{\frac{\partial}{\partial \theta_{1}}, \frac{\partial}{\partial \theta_{2}}\right\}$.

We can now address the smoothness of the moduli space.

Corollary 2.22. The moduli space of codimension $k$ foliations $\left.\mathrm{Fol}^{k}(M)\right) / \operatorname{Diff}_{0}(M)$ is generally not smooth.

Proof. We first remark that if a moduli space is smooth, then all its tangent spaces are locally isomorphic. Let $\lambda$ be a diophantine number, and consider the involutive distribution $K_{\lambda}$ on the 2 -torus as in Lemma 2.20 . If the moduli space was smooth nearby $\left[K_{\lambda}\right]$, it would be $1-$ dimensional, because the formal tangent space at $\left[K_{\lambda}\right]$ is 1-dimensional by Lemma 2.20. There is a sequence of rational numbers $\left(\lambda_{n}\right)$ converging to $\lambda$, since the rational numbers are dense in the real numbers. Thus we can find involutive distributions $K_{\lambda_{n}}$ arbitrarily close to $K_{\lambda}$ in the $C^{\infty}$-sense, and we know that the formal tangent space at each $\left[K_{\lambda_{n}}\right]$ is infinite-dimensional by Lemma 2.20 , thus not isomorphic to the one at $\left[K_{\lambda}\right]$. This provides a contradiction to the smoothness.

An alternative argument is as follows. If a moduli space is smooth nearby a given point $[K]$, then the deformations of $K$ are unobstructed. But we saw that this is not always the case, in Remark 2.21.

2.4.2. Moduli space of pre-symplectic structures. We know from Remark 2.11 that the formal tangent space to $\operatorname{Pre}^{-S_{y m}}{ }^{k}(M) / \operatorname{Diff}_{0}(M)$ at the equivalence class of a pre-symplectic form $\eta$ is

$$
T_{[\eta]}\left(\operatorname{Pre}_{-S_{y m}}^{k}(M) / \operatorname{Diff}_{0}(M)\right) \cong H_{\text {hor }}^{2}(M) \text {. }
$$

We show that its dimension can vary abruptly.

Recall the short exact sequence of complexes (1). It induces a long exact sequence in cohomology

$$
\cdots \rightarrow H^{1}(M) \rightarrow H^{1}(K) \stackrel{\delta}{\rightarrow} H_{\text {hor }}^{2}(M) \rightarrow H^{2}(M) \rightarrow \ldots
$$

where $H(K)$ denotes the foliated cohomology of the foliation $K$, where $H(M)$ is the de Rham cohomology of $M$, and $\delta$ is the connecting homomorphism. When $M$ is compact $t^{3}$ this leads to the following observation: $H^{1}(K)$ is infinite-dimensional iff $H_{\mathrm{hor}}^{2}(M)$ is infinite-dimensional.

Lemma 2.23. Let $M=\mathbb{T}^{3}$ be the 3-torus. For any $\lambda, \mu \in \mathbb{R}$ consider the pre-symplectic form

$$
\eta_{\lambda, \mu}:=d \theta_{2} \wedge d \theta_{3}-\lambda d \theta_{1} \wedge d \theta_{3}+\mu d \theta_{1} \wedge d \theta_{2} .
$$

Then

$$
\operatorname{dim}\left(H_{\text {hor }}^{2}(M)\right) \text { is } \begin{cases}\text { infinite } & \text { if }(\lambda, \mu) \in \mathbb{Q}^{2} \\ \text { finite } & \text { if }(1, \lambda, \mu) \text { satisfies the diophantine condition. }\end{cases}
$$

Above, following [2, Thm. 1.3.7] and its proof, we say that a vector $v \in \mathbb{R}^{3}$ satisfies the diophantine condition ${ }^{4}$ if there exists a positive real number $s$ such that inf $|m \cdot v|(|| m||)^{s}>0$, where the infimum ranges over all $m \in \mathbb{Z}^{3} \backslash\{0\}$ and the dot denotes the dot product in $\mathbb{R}^{3}$.

Proof. We make use of the observation just before the lemma. The kernel of $\eta_{\lambda, \mu}$ is the involutive distribution

$$
K_{\lambda, \mu}:=\operatorname{Span}\left\{\frac{\partial}{\partial \theta_{1}}+\lambda \frac{\partial}{\partial \theta_{2}}+\mu \frac{\partial}{\partial \theta_{3}}\right\}
$$

\footnotetext{
${ }^{3}$ This ensures that the de Rham cohomology of $M$ is finite-dimensional.

${ }^{4}$ Notice that the expression $|m \cdot v|(|| m||)^{s}$, specialized to the case $v=(1, \lambda) \in \mathbb{R}^{2}$, does not reduce exactly to the similar expression appearing just after Lemma 2.20 However, for fixed $\lambda$, the two diophantine conditions are equivalent (see [13, text after eq. (1.9)] for the statement, and [14, page 85] for a proof).
} 
If $(\lambda, \mu) \in \mathbb{Q}^{2}$, then $K_{\lambda, \mu}$ is the fiber distribution of a principal $U(1)$-bundle $M \rightarrow \mathbb{T}^{2}$, so $H^{1}\left(K_{\lambda, \mu}\right)$ is infinite-dimensional.

If $(1, \lambda, \mu)$ satisfies the diophantine condition, then $H^{1}\left(K_{\lambda, \mu}\right)$ is 1-dimensional. This is proven in [1] (see also [2, Thm. 1.3.7]), again using Fourier analysis.

Remark 2.24 (Obstructedness). In general, the deformation problem of pre-symplectic structures is formally obstructed. This was shown in [17, §6.1], refining the example mentioned for foliations in Remark 2.21

We finish addressing the smoothness of the moduli space.

Corollary 2.25. The moduli space of rank $k$ pre-symplectic structures $\operatorname{Pre}_{-S_{y m}}(M) / \operatorname{Diff}_{0}(M)$ is generally not smooth.

Proof. We just sketch the proof, since it is analogous to the one of Corollary 2.22. One approach is to use the fact that if a moduli space is smooth, then all its tangent spaces are locally isomorphic. For this, consider the pre-symplectic form $\eta_{\lambda, \mu}$ of Lemma 2.23 with $(1, \lambda, \mu)$ satisfying the diophantine condition, and notice that there is a sequence $\left(\lambda_{n}, \mu_{n}\right) \in \mathbb{Q}^{2}$ converging to $(\lambda, \mu)$.

Another approach is to use obstructed first order deformations, whose existence is guaranteed by Remark 2.24 .

\section{Appendix A. Pre-Symplectic forms on $M \times[0,1]$}

We present some elementary facts about pre-symplectic forms on a product manifold, which we use in Remark 2.19 and which are of independent interest. Any 2-form on $M \times[0,1]$ can be written as

$$
\Theta:=\eta_{t}+d t \wedge A_{t}
$$

for smooth families $\left(\eta_{t}\right)_{t \in[0,1]}$ in $\Omega^{2}(M)$ and $\left(A_{t}\right)_{t \in[0,1]}$ in $\Omega^{1}(M)$. To describe its kernel in terms of $\eta_{t}$ and $A_{t}$, one has to distinguish ${ }^{5}$ two cases:

$$
\operatorname{ker}(\Theta)_{(p, t)}= \begin{cases}\left\{v+c \partial_{t}: v \in T_{p} M, c \in \mathbb{R}, \iota_{v} \eta_{t}=-c A_{t}\right\} & \text { if } A_{t} \in\left(\operatorname{ker}\left(\eta_{t}\right)\right)^{\circ} \text { at } p \\ \operatorname{ker}\left(\eta_{t}\right) \cap \operatorname{ker}\left(A_{t}\right) & \text { otherwise. }\end{cases}
$$

From this we see that

$$
\operatorname{dim}\left(\operatorname{ker}(\Theta)_{(p, t)}\right)= \begin{cases}\operatorname{dim}\left(\operatorname{ker}\left(\eta_{t}\right)\right)+1 & \text { if } A_{t} \in\left(\operatorname{ker}\left(\eta_{t}\right)\right)^{\circ} \text { at } p \\ \operatorname{dim}\left(\operatorname{ker}\left(\eta_{t}\right)\right)-1 & \text { otherwise. }\end{cases}
$$

It can happen that $\Theta$ has constant rank and each of the two cases applies at points of $M \times[0,1]$ : take for instance $M=\mathbb{R}^{2}, \eta_{t}=t d x \wedge d y$ and $A_{t}=d x$, so that $\Theta=d x \wedge(t d y-d t)$.

Lemma A.1. Consider the 2-form $\Theta=\eta_{t}+d t \wedge A_{t}$ on $M \times[0,1]$. Any two of the following conditions implies the remaining one:

(1) $\Theta$ has constant rank

(2) for all $t$, the rank of $\eta_{t}$ is constant and independent of $t$

(3) the set of points $(p, t)$ for which $\left(A_{t}\right)_{p} \in\left(\operatorname{ker}\left(\eta_{t}\right)_{p}\right)^{\circ}$ is either $M \times[0,1]$ or empty.

Proof. Apply eq. (12).

Now we bring the closeness condition into play. Clearly a two form $\Theta=\eta_{t}+d t \wedge A_{t}$ is closed iff $d \eta_{t}=0$ and $\frac{\partial \eta_{t}}{\partial t}=d A_{t}$ for all $t$. Hence from Lemma A.1 we obtain:

\footnotetext{
${ }^{5}$ Indeed, $v+c \partial_{t} \in \operatorname{ker}\left(\eta_{t}+d t \wedge A_{t}\right)$ iff $\iota_{v} A_{t}=0$ and $\iota_{v} \eta_{t}=-c A_{t}$. In the case that $A_{t}$ lies in $\left(\operatorname{ker}\left(\eta_{t}\right)\right)^{\circ}=i m\left(\eta_{t}^{\sharp}\right)$, the second equation has solutions for all $c \in \mathbb{R}$ and the first equation is automatically satisfied.
} 
Corollary A.2. Consider smooth families $\left(\eta_{t}\right)_{t \in[0,1]}$ in $\Omega^{2}(M)$ and $\left(A_{t}\right)_{t \in[0,1]}$ in $\Omega^{1}(M)$. Assume that $A_{t} \in\left(\operatorname{ker}\left(\eta_{t}\right)\right)^{\circ}$ at every point. Then the following is equivalent:

- $\eta_{t}+d t \wedge A_{t}$ is a pre-symplectic form on $M \times[0,1]$

- for all $t, \eta_{t}$ is a pre-symplectic form on $M$ whose rank is independent of $t$, and $\frac{\partial \eta_{t}}{\partial t}=d A_{t}$.

\section{REFERENCES}

[1] J. L. Arraut and N. M. dos Santos. Linear foliations of $T^{n}$. Bol. Soc. Brasil. Mat. (N.S.), 21(2):189-204, 1991.

[2] M. Asaoka, A. El Kacimi Alaoui, S. Hurder, and K. Richardson. Foliations: dynamics, geometry and topology. Advanced Courses in Mathematics. CRM Barcelona. Birkhäuser/Springer, Basel, 2014.

[3] M. Crainic and I. Moerdijk. Deformations of Lie brackets: cohomological aspects. J. Eur. Math. Soc. (JEMS), 10(4):1037-1059, 2008.

[4] K. Fukaya. Deformation theory, homological algebra and mirror symmetry. In Geometry and physics of branes (Como, 2001), Ser. High Energy Phys. Cosmol. Gravit., pages 121-209. IOP, Bristol, 2003. www.mat .uniroma1.it/people/manetti/GeoSup0708/fukaya.pdf.

[5] A. Haefliger. Some remarks on foliations with minimal leaves. J. Differential Geometry, 15(2):269-284 (1981), 1980.

[6] J. L. Heitsch. A cohomology for foliated manifolds. Bull. Amer. Math. Soc., 79(6):1283-1285 (1974), 1973.

[7] J. Huebschmann. Higher homotopies and Maurer-Cartan algebras: quasi-Lie-Rinehart, Gerstenhaber, and Batalin-Vilkovisky algebras. In The breadth of symplectic and Poisson geometry, volume 232 of Progr. Math., pages 237-302. Birkhäuser Boston, Boston, MA, 2005.

[8] X. Ji. Simultaneous deformations of a Lie algebroid and its Lie subalgebroid. J. Geom. Phys., 84:8-29, 2014.

[9] P. P. La Pastina and L. Vitagliano. Deformations of linear Lie brackets. Pacific J. Math., 303(1):265-298, 2019.

[10] T. Lada and J. Stasheff. Introduction to SH Lie algebras for physicists. Internat. J. Theoret. Phys., 32(7):1087-1103, 1993

[11] J. M. Lee. Introduction to smooth manifolds, volume 218 of Graduate Texts in Mathematics. Springer, New York, second edition, 2013.

[12] C. C. Moore and C. L. Schochet. Global analysis on foliated spaces, volume 9 of Mathematical Sciences Research Institute Publications. Cambridge University Press, New York, second edition, 2006.

[13] J. Moser. A stability theorem for minimal foliations on a torus. Ergodic Theory and Dynamical Systems, $8: 251-281,121988$.

[14] J. Moser. Minimal foliations on a torus. Topics in calculus of variations, Lect. 2nd Sess., Montecatini/Italy 1987, Lect. Notes Math. 1365, 62-99, 1989.

[15] Y.-G. Oh and J.-S. Park. Deformations of coisotropic submanifolds and strong homotopy Lie algebroids. Invent. Math., 161(2):287-360, 2005.

[16] F. Schätz and M. Zambon. Deformations of pre-symplectic structures: a Dirac geometry approach. SIGMA, 14(128), 2018.

[17] F. Schätz and M. Zambon. Deformations of Pre-symplectic Structures and the Koszul $L_{\infty}$-algebra. International Mathematics Research Notices, 062018.

[18] L. Vitagliano. On the strong homotopy Lie-Rinehart algebra of a foliation. Commun. Contemp. Math., 16(6):1450007, 49 pages, 2014.

E-mail address: florian.schaetz@gmail.com

Eckenheimer Landstr. 136, 60318 Frankfurt am Main, Germany.

E-mail address: marco.zambon@kuleuven.be

Ku Leuven, Department of Mathematics, Celestijnenlaan 200B box 2400, 3001 Leuven, Belgium. 